\title{
Comparative anatomical analysis between the minipterional and supraorbital approaches
}

\author{
Rafael Martínez-Pérez, MD, PhD, ${ }^{1}$ Thiago Albonette-Felicio, MD, ${ }^{1}$ Douglas A. Hardesty, MD, ${ }^{1,2}$ and \\ Daniel M. Prevedello, MD1,2
}

Departments of ${ }^{1}$ Neurosurgery and ${ }^{2}$ Head and Neck Surgery, Wexner Medical Center, The Ohio State University, Columbus, Ohio

OBJECTIVE Keyhole approaches, namely the minipterional approach (MPTa) and the supraorbital approach (SOa), are alternatives to the standard pterional approach to treat lesions located in the anterior and middle cranial fossae. Despite their increasing popularity and acceptance, the indications and limitations of these approaches require further assessment. The purpose of the present study was to determine the differences in the area of surgical exposure and surgical maneuverability provided by the MPTa and SOa.

METHODS The areas of surgical exposure afforded by the MPTa and SOa were analyzed in 12 sides of cadaver heads by using a microscope and a neuronavigation system. The area of exposure of the region of interest and surgical freedom (maneuverability) of each approach were calculated.

RESULTS The area of exposure was significantly larger in the MPTa than in the SOa $\left(1250 \pm 223 \mathrm{~mm}^{2}\right.$ vs $939 \pm 139$ $\mathrm{mm}^{2}, \mathrm{p}=0.002$ ). The MPTa provided larger areas of exposure in the ipsilateral and midline compartments, whereas there was no significant difference in the area of exposure in the contralateral compartment. All targets in the anterior circulation had significantly larger areas of surgical freedom when treated via the MPTa versus the SOa.

CONCLUSIONS The MPTa provides greater surgical exposure and better maneuverability than that offered by the SOa. The SOa may be advantageous as a direct corridor for treating lesions located in the contralateral side or in the anterior cranial fossa, but the surgical exposure provided in the midline region is inferior to that exposed by the MPTa.

https://thejns.org/doi/abs/10.3171/2019.12.JNS193196

KEYWORDS aneurysm; skull base; minimally invasive; keyhole; pterional; anatomy; microsurgical; surgical technique

$\mathrm{M}$ INIMALLY invasive approaches (MIAs) have been widely accepted by the neurosurgical community for being a versatile and possibly less morbid alternative to the standard pterional craniotomy. ${ }^{9,32,35}$ Benefits derived from a reduced invasiveness include better cosmetic results, protection of the underlying brain parenchyma, shorter operating times, decreased risk of CSF leaks, and improvements in postoperative pain. ${ }^{34}$ These advantages, however, are meaningless if MIAs do not provide sufficient exposure to perform surgery safely and effectively.,21

Surgical case series have demonstrated the feasibility of using MIAs for approaching anterior circulation aneurysms and anterior and middle cranial fossae tumors. ${ }^{1-3}$, 9-11,15-17,19,23,24,27,30,33,34 In spite of increasing popularity and acceptance among neurosurgeons, cadaveric and anatomi- cal studies assessing the limits and feasibility of MIAs are sparse..$^{14,22}$ Clinical studies to date are mostly retrospective in nature, and most of them lack any randomization or pertinent statistical analysis. ${ }^{16,28}$ Hence, it is plausible that many of these clinical studies are biased toward good results. ${ }^{28}$

In vascular neurosurgery, the applicability of an MIA includes the minipterional approach (MPTa) and supraorbital approach (SOa) for the treatment of anterior circulation aneurysms. The treatment of middle cerebral artery (MCA) aneurysms is usually via the MPTa, whereas the SOa is usually preferred to treat anterior communicating artery (AComA) aneurysms. ${ }^{23,24}$ Nevertheless, such preferences are largely based on surgeon experience, rather than on any type of objective analysis.

The objective of this study, therefore, was to establish

ABBREVIATIONS $\mathrm{ACA}=$ anterior cerebral artery; $\mathrm{AComA}=$ anterior communicating artery; $\mathrm{ICA}=$ internal carotid artery; $\mathrm{MCA}=$ middle cerebral artery; $\mathrm{MIA}=$ minimally invasive approach; MPTa = minipterional approach; $\mathrm{PCA}=$ posterior cerebral artery; $\mathrm{PComA}=$ posterior communicating artery; $\mathrm{SOa}=$ supraorbital approach. SUBMITTED November 26, 2019. ACCEPTED December 30, 2019.

INCLUDE WHEN CITING Published online March 13, 2020; DOI: 10.3171/2019.12.JNS193196. 

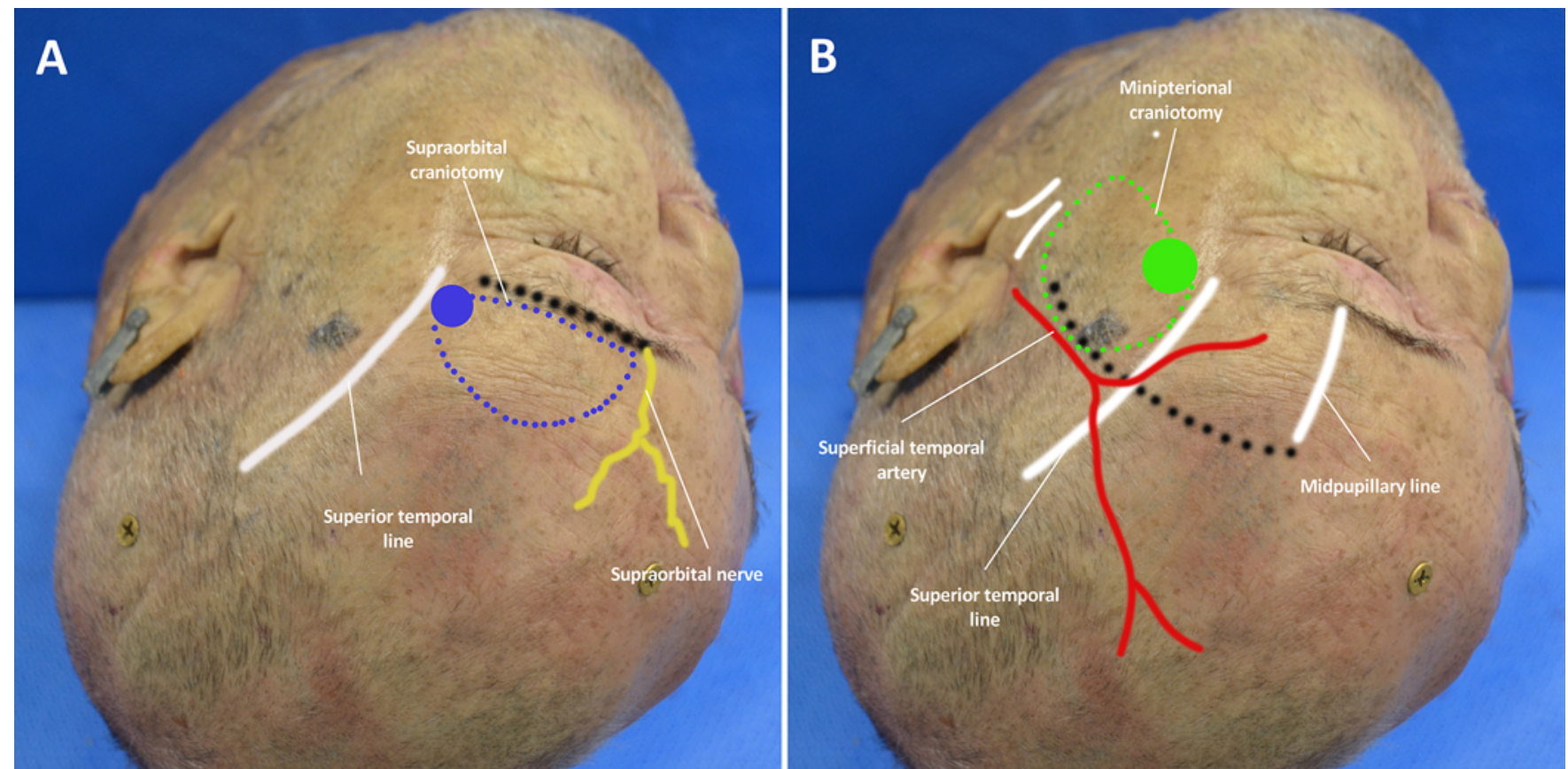

FIG. 1. Cadaveric specimens positioned simulating skin incision, surgical approach, and craniotomy limits in the $\mathrm{SOa}(\mathbf{A})$ and the MPTa (B). Figure is available in color online only.

indications and limits of both the MPTa and SOa techniques in the treatment of intracranial aneurysms from the anterior circulation and anterior and middle fossae lesions, through a pertinent comparative anatomical study with modern analytical techniques.

\section{Methods}

Anatomical cadaveric dissections and pertinent measurements were performed using standard institutionally approved practices for cadaveric specimens at the Anatomical Laboratory for VisuoSpatial Innovations in Otolaryngology and Neurosurgery (ALT-VISION) at The Ohio State University. Prior to dissections, 6 fixed human cadaveric heads were prepared and injected with red silicone through the common carotid and vertebral arteries and with blue silicone through the jugular veins.

The methodology regarding the acquisition of reference points for neuronavigation and measurements was similar to that used in previous works. ${ }^{18}$ Briefly, cadaveric heads were scanned using high-resolution CT imaging. Images were uploaded to the iNtellect Cranial Navigation System (Stryker). Cadaveric heads were registered in the neuronavigation system by using surface recognition with the BrainLab Curve for the acquisition of landmark points for the operative exposure calculation. Surface-matching refinement based on bone surface was additionally performed, in order to obtain an error of less than $0.5 \mathrm{~mm}$ for all specimens.

Cadaveric heads were positioned slightly hyperextended, rotated $20^{\circ}-30^{\circ}$ contralateral to the side of the approach (Fig. 1). Both MPTa and SOa were performed using drills (Stryker-Leibinger Corp./Medtronic) and macroscopic and microscopic dissection tools (KLS Mar- tin Group). Microsurgical intradural dissections were performed under microscope visualization (Carl Zeiss Co.).

\section{Surgical Technique}

For the SOa (Fig. 2A-C), we used the same approach as initially described by Perneczky and colleagues..$^{13,27,32}$ An eyebrow incision was made starting immediately lateral to the level of the supraorbital notch. The periosteum was retracted inferiorly and a $2 \times 3-\mathrm{cm}$ craniotomy performed. No orbital rim removal was used. The base of the anterior cranial fossa was flattened using drills before dural opening. In specimens with prominent pneumatization we entered the frontal sinus in order to prevent a false decrease in the overall surgical exposure. Thereafter, a Cshaped dural incision was performed. Opening the arachnoid membranes between the frontal lobe and the cranial base allowed gravity to move the frontal lobe away and let the operator use the subfrontal corridor to access the anterior wall of both optic nerves and internal carotid arteries (ICAs). Further dissection of the opticocarotid, suprasellar, and prechiasmatic cisterns provided exposure of the AComA, the proximal segment of both posterior communicating arteries (PComAs), and the MCAs. Finally, the durotomy was closed using 3-0 silk sutures and the bone flap was replaced using miniplates. After closure, the MPTa was performed and measured on the same specimens.

The MPTa procedure (Fig. 2D-F) has been widely published elsewhere and explained in depth. ${ }^{3,9,18-20}$ A curvilinear frontal incision extending from a line situated $1 \mathrm{~cm}$ above the zygoma to the midpupillary line was performed. The skin flap was anteriorly retracted and an interfascial dissection of the temporalis muscle was performed in or- 

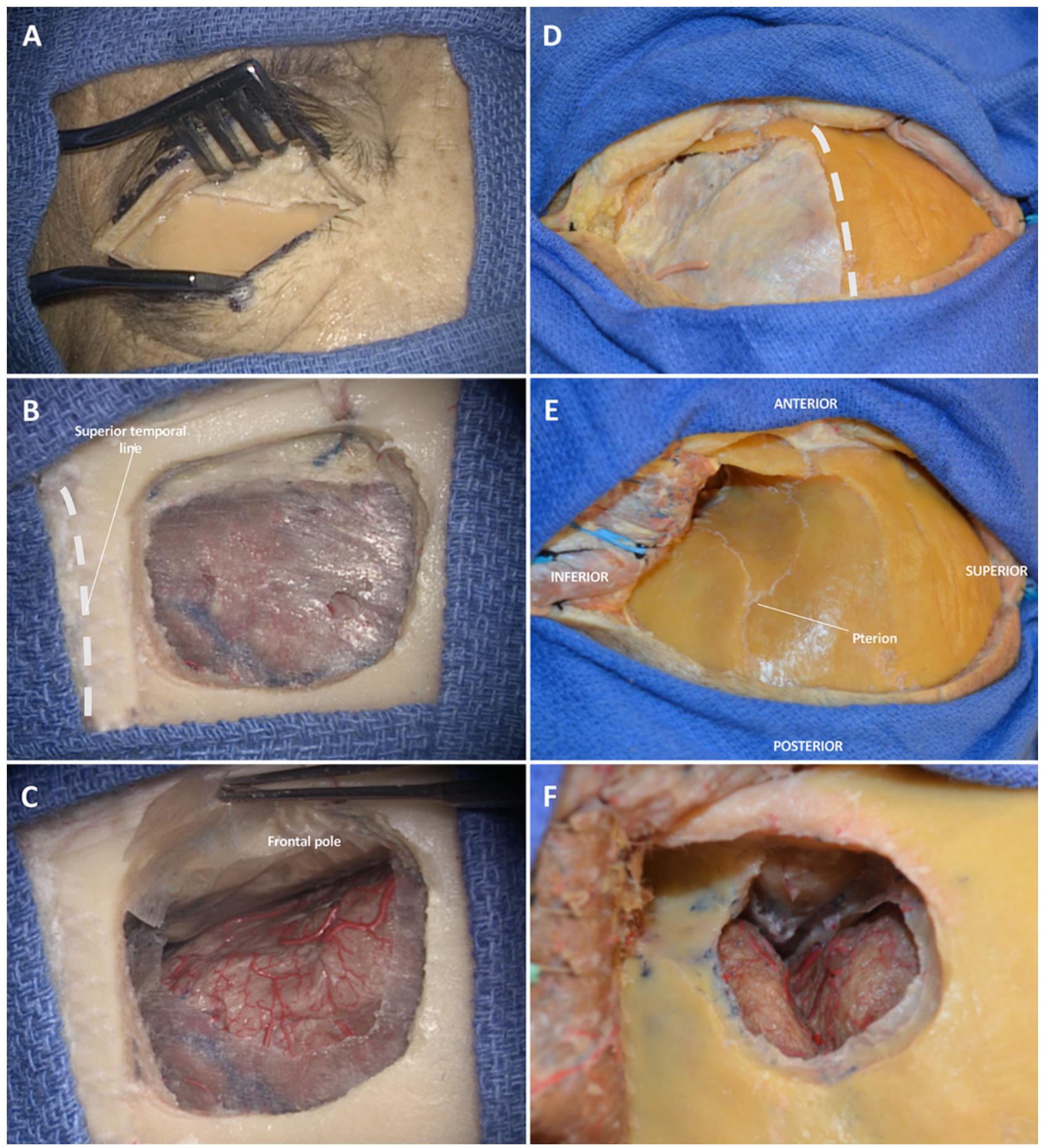

FIG. 2. Stepwise dissection (skin incision and craniotomy) in the SOa (A-C) and the MPTa (D-F). The skin incision for the SOa is placed lateral to the supraorbital notch and the subcutaneous tissue is sharply dissected until the superior temporal line and the temporalis fascia are exposed (A). A $3 \times 2-\mathrm{cm}$ craniotomy above the orbital rim and lateral to the supraorbital notch is performed (B). The dura is incised in a curvilinear fashion with its base directed toward the orbit (C). For the MPTa, after the skin incision, an interfascial temporalis muscle dissection is required to expose the orbital rim and retract the temporalis mucle inferiorly (D). A 3 $\times 3-\mathrm{cm}$ minipterional craniotomy located beneath the superior temporal line is performed. The pterion marks the posterior limit of the craniotomy $(\mathrm{E})$. The pterion is an important bony landmark, because it corresponds intracranially with the anterior sylvian point $(F)$. Figure is available in color online only.

der to expose the superior end of the orbital rim. The temporalis muscle was then subperiosteally dissected and inferiorly retracted until the squamous suture was exposed.
A $3 \times 3-\mathrm{cm}$ minipterional craniotomy located beneath the superior temporal line and anterior to the pterion was performed. At this point, the sphenoid ridge was drilled until 


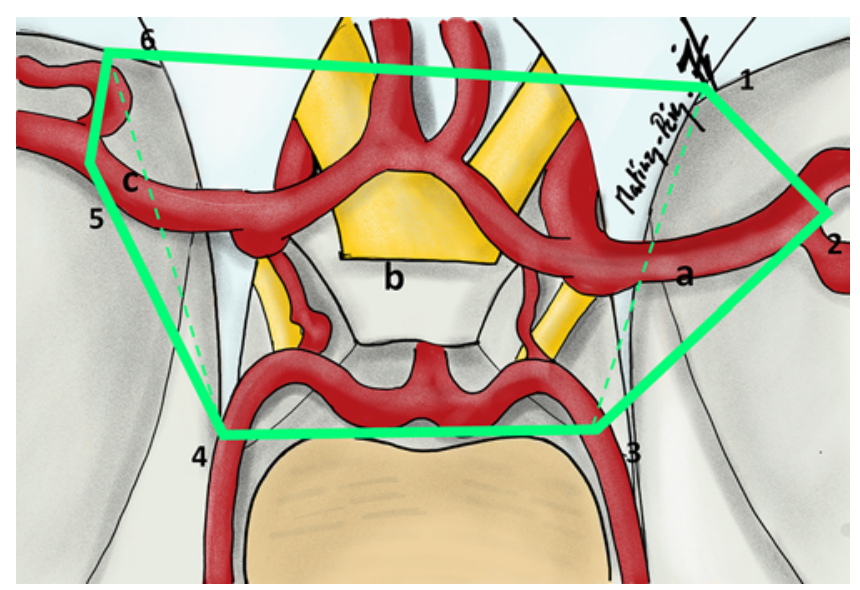

FIG. 3. Schematic drawing delineating the region of interest defined for calculations of the area of exposure in this work. The total area of exposure was defined by a hexagonal region framed by the following points: 1) a point located lateral to the superior orbital fissure; 2) the ipsilateral MCA bifurcation; 3 ) the most distal point of the ipsilateral PCA; 4) the most distal point of the contralateral PCA; 5 ) the most distal point of the contralateral MCA; and 6) the most distal point on the contralateral sphenoid ridge that is surgically reachable through the pertinent approach. Additionally, the total area of exposure was subclassified into 3 smaller regions: a) the ipsilateral paramedian region (framed by points 1,2 , and 3 ); $\mathbf{b}$ ) the central or midline region (framed by points $1,3,4$, and 6); and c) the contralateral paramedian region (framed by points 4 , 5, and 6). Copyright Rafael Martínez-Pérez. Published with permission. Figure is available in color online only.

the meningoorbital band was exposed and the superior orbital fissure was reached. The meningoorbital band was sectioned and an interdural dissection was performed in a similar way to that described by Hakuba and colleagues. ${ }^{12}$ Thereafter, the dura mater was opened in C-shaped fashion with the base directed anteriorly toward the orbit. Once at the intradural space, a sylvian dissection in front of the anterior sylvian point was performed. After exposing the proximal compartment of the sylvian cistern, the ipsilateral opticocarotid, chiasmatic, and crural cisterns were dissected in order to expose the ICA, MCA, anterior cerebral artery (ACA), AComA, and PComA. After dissection, stereotactic measurements for the MPTa were obtained.

\section{Measurements}

Methods for calculating area of exposure and surgical freedom were previously described by this group..$^{18}$ Neuronavigation was used to obtain the coordinates in 3 axes of each point. Then, all landmark coordinates were grouped and processed using a dedicated software (Microsoft Office Excel 2013) that calculates an area from a spreadsheet of $3 \mathrm{D}$ coordinates.

\section{Area of Exposure}

The area of exposure was calculated using the same reference points that were previously used by Figueiredo et al. ${ }^{9}$ Such references were selected in order to include the most representative points of the anterior circulation.

The total area of exposure of the region of interest was defined as that limited by the following 6 points (Fig. 3): 1) the ipsilateral sphenoid ridge, just lateral to the superior orbital fissure; 2) the ipsilateral MCA bifurcation; 3) the most distal point of the posterior cerebral artery (PCA); 4) the most distal point of the contralateral PCA; 5) the most distal point of the MCA; and 6) the most lateral point of the contralateral sphenoid ridge. In cases in which the contralateral PCA could not be exposed, the most distal point of the contralateral PComA is referenced as point 5 of the area of exposure.

Additionally, the total area of exposure was divided into 3 regions as follows (Fig. 3): a) ipsilateral paramedian region-the triangular space limited by points 1,2 , and 3 ; b) midline region - the quadrangular space limited by points $1,3,4$, and 6 ; and c) contralateral paramedian region-the triangular space limited by points 4,5 , and 6 .

\section{Surgical Freedom}

Surgical maneuverability was assessed by means of the surgical freedom following the method described in previous works. ${ }^{6} 18$ Surgical freedom was defined as the area delineated by the tip of a $25-\mathrm{cm}$ straight dissector reaching the extreme position in 2 perpendicular axes (4 points), while holding the proximal tip on the selected target of interest. Surgical freedom was calculated at the same target points selected by Figueiredo et al. ${ }^{9}$ These target points were located at 1) the ipsilateral bifurcation of the MCA; 2) the ipsilateral bifurcation of the ICA; and 3) the AComA. Such points of interests were selected to characterize the most representative surgical targets in the anterior circulation so that the exposure of these structures in both approaches could be evaluated.

\section{Statistical Analysis}

Differences in "area of exposure" and "surgical freedom" between the MPTa and SOa were analyzed using parametric tests (Student t-test). A p value $<0.05$ was considered significant. All tests were calculated using RStudio version 1.0.136. The mean values are expressed \pm SD.

\section{Results}

Examples of the intradural view of the MPTa and the $\mathrm{SOa}$ are represented in Fig. 4. The ipsilateral MCA and ICA were exposed in all specimens for both approaches. The ipsilateral PCA was exposed in all but 1 side for both approaches. The contralateral PCA was exposed in 10 of 12 sides when using an SOa and in 8 of 12 sides when using an MPTa.

\section{Area of Exposure}

The total area of exposure was significantly higher for the MPTa $\left(1250 \pm 223 \mathrm{~mm}^{2}\right)$ than for the SOa $(939 \pm 139$ $\mathrm{mm}^{2}$, independent-sample t-test, $\mathrm{t}=3.74, \mathrm{p}=0.002, \mathrm{~d}=$ 15.1) (Table 1, Fig. 5). The area of exposure in the ipsilateral paramedian region and in the midline region was also significantly larger for the MPTa than for the SOa (paramedian $192 \pm 39.9 \mathrm{~mm}^{2}$ vs $125 \pm 39.3 \mathrm{~mm}^{2}, \mathrm{t}=3.78$, $\mathrm{p}=$ $0.001, \mathrm{~d}=18$; midline $963 \pm 180 \mathrm{~mm}^{2}$ vs $755 \pm 114 \mathrm{~mm}^{2}$, $\mathrm{t}=3.09, \mathrm{p}=0.007, \mathrm{~d}=15.2$ ). No significant differences 

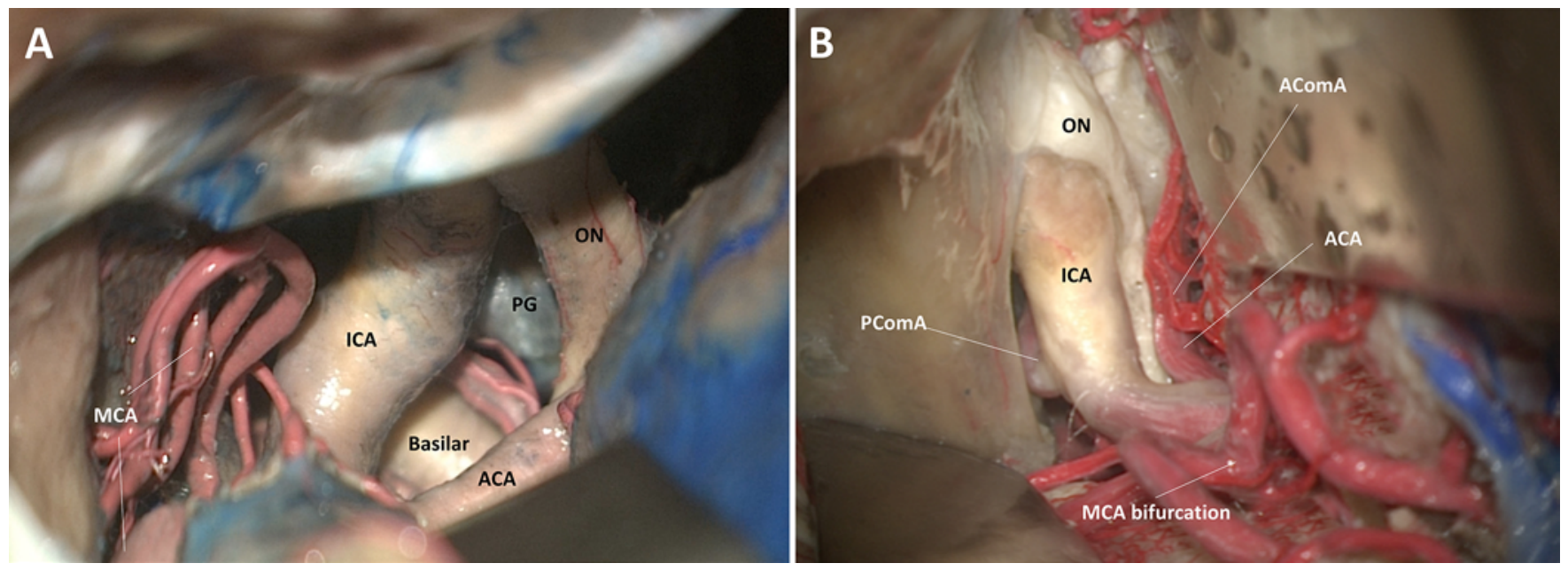

FIG. 4. Surgical view of the intradural space provided by the SOa (A) and the MPTa (B). A: SOa, left side. A subfrontal corridor is used to get exposure of both optic nerves and intracranial ICAs from an anterior and superior perspective. B: MPTa, left side. Exposure of the anterior sylvian point is extremely important in this approach. Sylvian dissection proximal to this point provides the largest anatomical exposure and the optimal possibility of minimizing brain retraction. After sylvian and arachnoid dissection, the ICA, optic nerves, ACA, and MCA are well visualized and exposed. Bas = basilar artery; ON = optic nerve; $P G=$ pituitary gland. Figure is available in color online only.

were found between the area of exposure in the contralateral paramedian region provided by the MPTa $(95.5 \pm$ $\left.56.2 \mathrm{~mm}^{2}\right)$ and that provided by the SOa $\left(59.4 \pm 38.9 \mathrm{~mm}^{2}\right.$, $\mathrm{t}=1.67, \mathrm{p}=0.11, \mathrm{~d}=16)$.

\section{Surgical Freedom}

Surgical freedom provided by the MPTa was significantly larger than that provided by the $\mathrm{SOa}$ at the ICA bifurcation $\left(15.1 \pm 4.9 \mathrm{~mm}^{2}\right.$ vs $2.8 \pm 1.1 \mathrm{~mm}^{2}, \mathrm{t}=7.6, \mathrm{p}<$ $0.001, \mathrm{~d}=9.9)$; at the MCA $\left(21.5 \pm 5.8 \mathrm{~mm}^{2}\right.$ vs $2.8 \pm 2.8$ $\left.\mathrm{mm}^{2}, \mathrm{t}=9.2, \mathrm{p}<0.001, \mathrm{~d}=13\right)$; and at the AComA (10.1 $\pm 4.7 \mathrm{~mm}^{2}$ vs $3.1 \pm 0.8 \mathrm{~mm}^{2}, \mathrm{t}=4.6, \mathrm{p}=0.001, \mathrm{~d}=9.6$ ) (Table 2, Fig. 6).

\section{Discussion}

We demonstrate that the MPTa affords a larger area of exposure and better surgical maneuverability in anterior and middle fossae when compared to the SOa craniotomy. These differences are particularly accentuated for targets located in the ipsilateral and midline compartment. Conversely, the MPTa was not superior to the SOa in the surgical exposure of the contralateral compartment.

Although the size of the craniotomy is similar between the two approaches, each yields significantly different viewing angles. ${ }^{7,24}$ This provides a quantitative rationale supporting previous works in which one approach was used over the other in certain clinical lesions. ${ }^{28,29}$ The surgical view provided by the $\mathrm{SOa}$ exposes from anteriorly the AComA complex and both optic nerves, following a subfrontal trajectory. ${ }^{24} \mathrm{~A}$ recent systematic review found that AComA lesions were the most frequently reported aneurysm type treated via an SOa. ${ }^{29}$ Some authors have discussed the superiority of the SOa for treating midline and contralateral lesions, given the shorter distance from the craniotomy to the target. ${ }^{2,16,17,24}$ Tra et al. ${ }^{30}$ supported the idea that the $\mathrm{SOa}$ is an excellent alternative for clip- ping AComA and contralateral aneurysms, due to its superiority over the MPTa in terms of viewing and working angles. For similar reasons, the SOa has been generally selected for the treatment of bilateral mirror aneurysms. ${ }^{13,17}$, ${ }^{23,33}$ Moreover, the aneurysm projection and its relationship with the working angle also determine the degree of complexity and the amount of dissection that is required to expose the aneurysm. As such, for microsurgical clipping the most straightforward corridor is the one whose plane is orthogonal with the neck of the aneurysm, so this can be exposed without the need to dissect the aneurysm dome. For instance, the SOa could be a suitable option not only for AComA aneurysms, but also for those PComA aneurysms that are pointing laterally, and ICA bifurcation aneurysms projected superiorly. ${ }^{27,28,32}$

These previous clinical observations conflict, however, with our current anatomical results. The SOa has a limited area of exposure in the midline compartment, and a reduced maneuverability for all 3 targets analyzed in the anterior circulation, including the AComA and the ICA bifurcation. Although the SOa is a direct approach to midline lesions, some drawbacks explain our current quantitative findings of lessened area of exposure and maneuver-

TABLE 1. Area of exposure provided by the MPTa and the SOa

\begin{tabular}{lccc}
\hline \multirow{2}{*}{ Area of Interest } & \multicolumn{2}{c}{ Area of Exposure $\left(\mathrm{mm}^{2}\right)$} & \\
\cline { 2 - 3 } & MPTa & SOa & p Value \\
\hline Total & $1250 \pm 223$ & $939 \pm 139$ & 0.002 \\
\hline Ipsilat paramedian & $192 \pm 39.9$ & $125 \pm 39.3$ & 0.001 \\
\hline Midline & $963 \pm 180$ & $755 \pm 114$ & 0.007 \\
\hline Contralat paramedian & $95.5 \pm 56.2$ & $59.4 \pm 38.9$ & 0.11 \\
\hline
\end{tabular}

Values are expressed as the mean $\pm S D$. The $p$ values were calculated according to independent-sample t-test. 


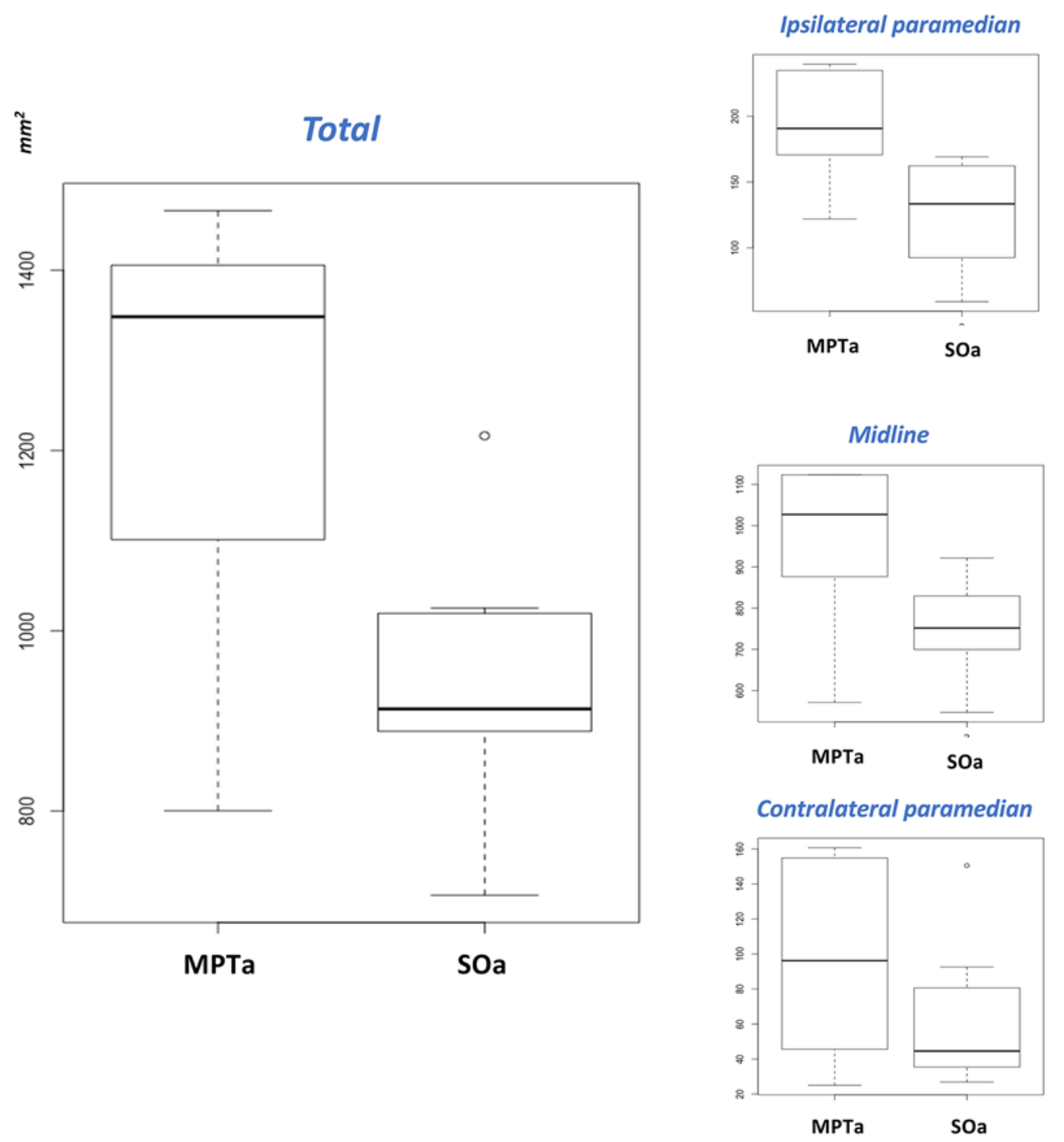

FIG. 5. Differences between the MPTa and the SOa in the area of exposure along different regions included in the analysis. Comparisons are displayed in boxplots. The boxplot's depth provides an idea of the sample dispersion; each extreme represents the 25th and 75th percentile, whereas the horizontal line represents the median, and extremes of the perpendicular line denote the range of the sample. Figure is available in color online only.

ability or surgical freedom. The lateral limit of the SOa craniotomy, located at the level of the superior temporal line, obstructs access to the sylvian fissure. This means that further frontal lobe retraction is needed to provide sufficient exposure of the surgical target when using the subfrontal corridor, even for approaching the anterior cranial fossa and midline region., ${ }^{1,9}$

Moreover, because the dissection of the sylvian fissure is hindered when using an SOa, surgical maneuverability in the middle cranial fossa is also reduced. Some authors have previously shown technical difficulties in approaching middle fossa lesions via an SOa. ${ }^{7,15,28,29}$ Kang et al. ${ }^{15}$ proved that viewing angles provided by the SOa were lower when approaching MCA aneurysms, especially if the aneurysm was located below the lesser sphenoid wing. Nevertheless, beyond these clinical series, limitations of the SOa in accessing the middle cranial fossa have not previously been quantitatively evaluated..$^{15,28,29}$ Our present study demonstrated a $35 \%$ decrease in the surgical exposure of the ipsilateral compartment if an SOa is used, when compared to that provided by MPTa.

The MPTa craniotomy is performed beneath the superior temporal line. This requires temporalis muscle dissection beyond that of the SOa, but unlike the SOa it also affords proximal exposure of the sylvian fissure. Sylvian splitting proximal to the anterior sylvian point provides additional brain relaxation and retraction, and increases the surgical maneuverability, especially in the middle fossa. ${ }^{4,5,9}$ The exposure of the frontal lobe is reduced in comparison to the traditional pterional craniotomy, because the superior aspect of the MPTa is limited by the superior temporal line. As a consequence, the use of the subfrontal 
TABLE 2. Surgical freedom for each surgical target provided by the MPTa and the SOa

\begin{tabular}{lccc}
\hline & \multicolumn{2}{c}{ Surgical Freedom $\left(\mathrm{mm}^{2}\right)$} & \\
\cline { 2 - 3 } Target of Interest & MPTa & SOa & p Value \\
\hline ICA bifurcation & $15.1 \pm 4.9$ & $2.8 \pm 1.1$ & $<0.001$ \\
\hline MCA bifurcation & $21.5 \pm 5.8$ & $2.8 \pm 2.8$ & $<0.001$ \\
\hline AComA & $10.1 \pm 4.7$ & $3.1 \pm 0.8$ & 0.001 \\
\hline
\end{tabular}

Values are expressed as the mean $\pm S D$. The $p$ values were calculated according to independent-sample t-test.

corridor is impeded and some consider this a limitation to properly approaching lesions in the anterior cranial fossa and midline region. ${ }^{5}$ Our current anatomical analysis does not support this concern; we found excellent exposure and maneuverability for targets in both areas. Indeed, the use of the transsylvian corridor, enhanced by the lateral mobilization of the temporal lobe, permits a wider exposure of the midline compartment while reducing brain manipulation..$^{14,15}$

Our results are supported by at least one prior clinical series showing excellent results in clipping any type of anterior circulation aneurysm using the MPTa. ${ }^{9,10,34}$ Figueiredo and colleagues popularized the MPTa craniotomy combined with the interfascial dissection technique as a modification of the traditional pterional craniotomy. As in the transorbital approach, they used an intraduralsubfrontal-transsylvian approach to reach lesions located in the anterior and middle cranial fossae. Our MPTa is modified somewhat to more of a skull base approach, ${ }^{3,19}$ which, in contrast to the original MPTa, ${ }^{9}$ uses the extra- dural pretemporal route to obtain additional exposure. Drilling of the sphenoid ridge and an interdural dissection of the middle fossa dura allows mobilization of the temporal lobe without significant brain retraction and provides access to the lateral wall of the cavernous sinus and paraclinoid region. Furthermore, most paraclinoid region aneurysms are also suitable for clipping using the MPTa technique if combined with an anterior clinoidectomy, providing at the same time a wide view of the aneurysm and optic nerve decompression. ${ }^{3,19}$

Besides the conclusions derived from the anatomical analysis, there are other key factors that were not included in our study and are worth discussing when considering the use of an MPTa. Therefore, we concur with Eroglu and colleagues, who considered that MIAs are relevant not just for offering smaller incisions and reduced operating times, but also for providing the most direct corridor to the target with the least possible cerebral disruption., ${ }^{7,21}$ In this sense, the SOa affords the shortest distance to lesions located in the anterior cranial fossa, such as tuberculum sella or planum sphenoidale meningiomas. In these cases, just the dissection of the arachnoid membranes between the frontal lobe and the cranial base is enough to access the ventral face of the lesion. Hence, the improvement in the area of exposure and maneuverability alone by the MPTa does not justify its use in these pathologies. ${ }^{7,25}$ Conversely, the MPTa has been reported to be a very straightforward craniotomy for approaching aneurysms located in the $\mathrm{MCA}^{8,28}$ or extradural tumors located in the middle cranial fossa (e.g., middle fossa meningiomas, chondromas, trigeminal schwanommas). ${ }^{19,31}$

In addition, the relationship between the optic chiasm and a midline lesion is also a determinant for approach

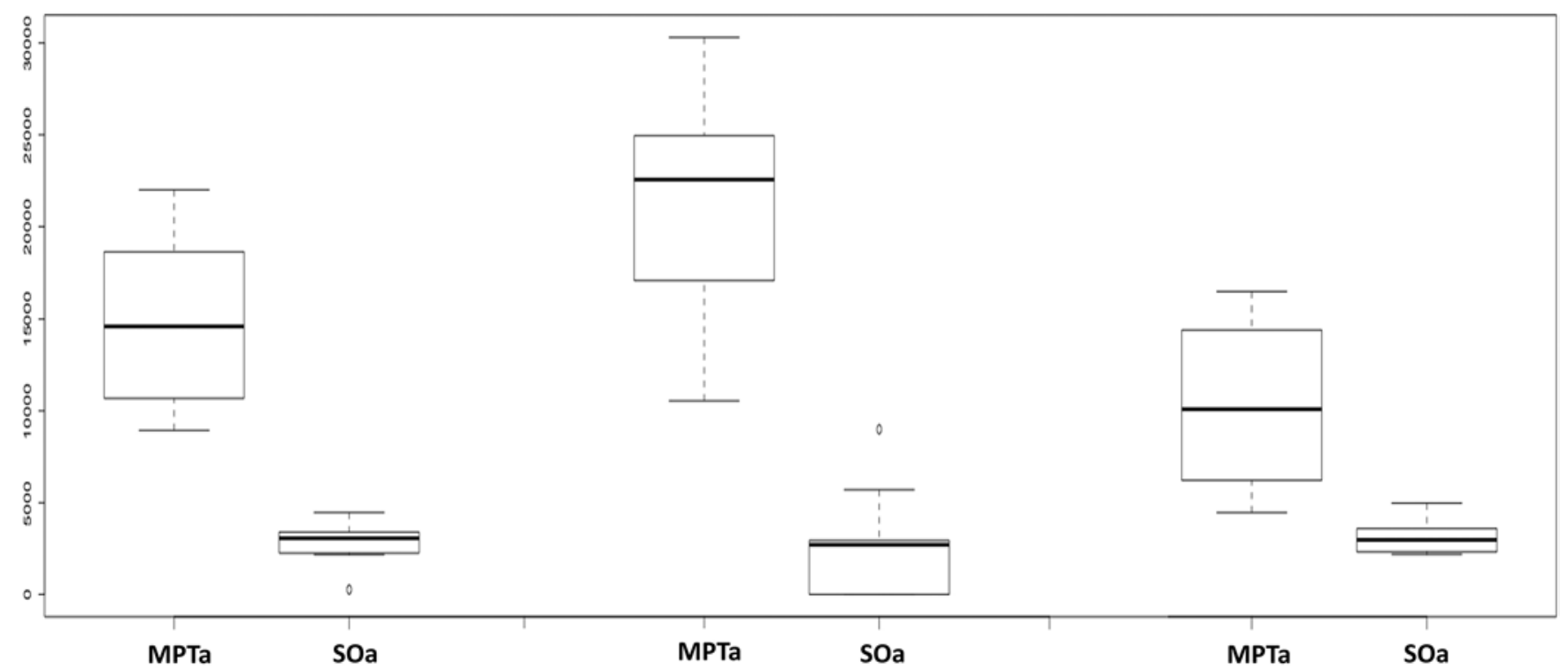

FIG. 6. Differences in the surgical freedom between the MPTa and the SOa for each target of interest. Comparisons are displayed in boxplots. The boxplot's depth provides an idea of the sample dispersion; each extreme represents the 25th and 75th percentile, whereas the horizontal line represents the median, and extremes of the perpendicular line denote the range of the sample. 
selection. In cases of prefixed chiasms or when the optic apparatus is displaced superiorly by the tumor, as in some tuberculum sella meningiomas, the SOa does not represent such a good alternative and an MPTa could be regarded as a safer option. This means that regardless of the surgical exposure provided by each approach, we can conclude that the SOa is a reasonable alternative for lesions located above the sphenoid ridge and anterior to the optic nerve, whereas the MPTa is an excellent approach for lesions located below these landmarks. ${ }^{25}$

We must acknowledge some limitations in the present work. First, the region of interest defined to assess the area of exposure included the most relevant targets on the anterior circulation that can be reached via a pterional approach as proposed by Figueiredo and colleagues. ${ }^{9} \mathrm{Be}-$ cause the MPTa is considered a variant of the pterional approach, with more similarities than the SOa in terms of the angle of attack and working angle, it might be thought that this study could be potentially biased toward the benefit of the MPTa. Selection of another region of interest along the skull base might have derived different results. For instance, a region of interest entirely located in the anterior cranial fossa would have benefitted the area of exposure to the detriment of the MPTa, and vice versa if the region of interest was located in the middle cranial fossa. However, we should reinforce the fact that the decision in choosing such regions of interest was based on a previous literature review of anatomical studies with similar objectives to the present study. By proceeding on this basis, results of the present work can be corroborated and compared with those reported by others, and hence the external validity is also improved. Still, it would be interesting to conduct further anatomical studies assessing differences in the surgical exposure and maneuverability for approaching other regions (e.g., interpeduncular and prepontine region, posterior circulation, anterior cranial fossa).

Some authors have suggested that vascular lesions and aneurysms cannot be safely treated using MIAs without endoscopic assistance. ${ }^{22,26,30}$ However, all dissections in our study were carried out using only microsurgical techniques, and adequate exposure and freedom were obtained without undue difficulty.

In addition to the other limitations, cadaveric anatomical specimens are stiffer than in vivo human tissues. Consequently, and due to the lack of brain retraction, the area of exposure and surgical freedom values could be underestimated. Nevertheless, our results support the possibility of drawing some conclusions that can help the surgeon to select the most appropriate approach based on a systematic analysis of the surgical exposure and maneuverability provided by each technique. A prospective analysis of surgical utilization is necessary to assess the burden of potential real-life complications. An MIA does possess some advantages over other traditional approaches, such as the pterional craniotomy. However, the neurosurgeon should recognize the limitations for each approach and the pitfalls that can lead to complications. We do not recommend a rigid use of one single approach. Indeed, we believe that optimal outcomes are achieved when the appropriate approach is selected for an individual patient and pathology.

\section{Conclusions}

In comparison to the SOa, the MPTa provides larger areas of exposure and surgical maneuverability for lesions located in the middle cranial fossa. Although the SOa is a more direct route to the anterior cranial fossa, the MPTa in our study provided superior surgical exposure or maneuverability to most common clinical targets. The MPTa also offers an excellent exposure of the midline region by using the transsylvian and pretemporal corridors.

\section{Acknowledgments}

Dissections and further calculations were performed at the Anatomical Laboratory for VisuoSpatial Innovations in Otolaryngology and Neurosurgery (ALT-VISION) at The Ohio State University, adhering to the previously institutionally approved practices for cadaveric specimens.

\section{References}

1. Andrade-Barazarte H, Jägersberg M, Belkhair S, Tymianski $\mathrm{R}$, Turel MK, Schaller K, et al: The extended lateral supraorbital approach and extradural anterior clinoidectomy through a frontopterio-orbital window: technical note and pilot surgical series. World Neurosurg 100:159-166, 2017

2. Caplan JM, Papadimitriou K, Yang W, Colby GP, Coon AL, Olivi A, et al: The minipterional craniotomy for anterior circulation aneurysms: initial experience with 72 patients. Neurosurgery 10 (Suppl 2):200-207, 2014

3. Chiarullo M, Mura J, Rubino P, Rabelo NN, Martinez-Perez R, Figueiredo EG, et al: Technical description of minimally invasive extradural anterior clinoidectomy and optic nerve decompression. World Neurosurg 129:e502-e513, 2019

4. Cohen-Gadol AA: Atraumatic sylvian fissure split: nuances and pitfalls. Oper Neurosurg (Hagerstown) 18:217-224, 2020

5. Davies JM, Lawton MT: Advances in open microsurgery for cerebral aneurysms. Neurosurgery 74 (Suppl 1):S7-S16, 2014

6. Elhadi AM, Hardesty DA, Zaidi HA, Kalani MYS, Nakaji P, White WL, et al: Evaluation of surgical freedom for microscopic and endoscopic transsphenoidal approaches to the sella. Neurosurgery 11 (Suppl 2):69-79, 2015

7. Eroglu U, Shah K, Bozkurt M, Kahilogullari G, Yakar F, Dogan I, et al: Supraorbital keyhole approach: lessons learned from 106 operative cases. World Neurosurg 124:e667-e674, 2019

8. Esposito G, Dias SF, Burkhardt JK, Fierstra J, Serra C, Bozinov O, et al: Selection strategy for optimal keyhole approaches for middle cerebral artery aneurysms: lateral supraorbital versus minipterional craniotomy. World Neurosurg 122:e349-e357, 2019

9. Figueiredo EG, Deshmukh P, Nakaji P, Crusius MU, Crawford N, Spetzler RF, et al: The minipterional craniotomy: technical description and anatomic assessment. Neurosurgery 61 (5 Suppl 2):256-265, 2007

10. Figueiredo EG, Teixeira MJ, Spetzler RF, Preul MC: Clinical and surgical experience with the minipterional craniotomy. Neurosurgery 75:E324-E325, 2014

11. Figueiredo EG, Welling LC, Preul MC, Sakaya GR, Neville I, Spetzler RF, et al: Surgical experience of minipterional craniotomy with 102 ruptured and unruptured anterior circulation aneurysms. J Clin Neurosci 27:34-39, 2016

12. Hakuba A, Nishimura S, Shirakata S, Tsukamoto M: [Surgical approaches to the cavernous sinus. Report of 19 cases (author's transl).] Neurol Med Chir (Tokyo) 22:295-308, 1982 [Japanese]

13. Hopf NJ, Stadie A, Reisch R: Surgical management of bilat- 
eral middle cerebral artery aneurysms via a unilateral supraorbital key-hole craniotomy. Minim Invasive Neurosurg 52:126-131, 2009

14. Jägersberg M, Brodard J, Qiu J, Mansouri A, Doglietto F, Gentili F, et al: Quantification of working volumes, exposure, and target-specific maneuverability of the pterional craniotomy and its minimally invasive variants. World Neurosurg 101:710-717.e2, 2017

15. Kang HJ, Lee YS, Suh SJ, Lee JH, Ryu KY, Kang DG: Comparative analysis of the mini-pterional and supraorbital keyhole craniotomies for unruptured aneurysms with numeric measurements of their geometric configurations. J Cerebrovasc Endovasc Neurosurg 15:5-12, 2013

16. Lan Q, Zhang H, Zhu Q, Chen A, Chen Y, Xu L, et al: Keyhole approach for clipping intracranial aneurysm: comparison of supraorbital and pterional keyhole approach. World Neurosurg 102:350-359, 2017

17. Martellotta N, Gigante N, Toscano S, Maddalena GF, Tripodi M, Settembrini G, et al: Unilateral supraorbital keyhole approach in patients with middle cerebral artery (M1-M2 segment) symmetrical aneurysms. Minim Invasive Neurosurg 46:228-230, 2003

18. Martínez-Pérez R, Albonette-Felicio T, Zachariah MA, Hardesty DA, Carrau RL, Prevedello DM: Quantitative anatomic study of the minipterional craniotomy in the paraclinoid region: benefits of the extradural anterior clinoidectomy. World Neurosurg [epub ahead of print], 2019

19. Martínez-Pérez R, Hernández-Álvarez V, Maturana R, Mura JM: The extradural minipterional pretemporal approach for the treatment of spheno-petro-clival meningiomas. Acta Neurochir (Wien) 161:2577-2582, 2019

20. Martinez-Perez R, Joswig H, Albiña P, Perales I, Poblete T, Mura J: The extradural minipterional approach for the treatment of paraclinoid aneurysms: a cadaver stepwise dissection and clinical case series. Neurosurg Rev 43:361-370, 2020

21. Martinez-Perez R, Mura JM: The extradural minipterional approach: "think small, play wider". World Neurosurg 125:534-535, 2019

22. Noiphithak R, Yanez-Siller JC, Revuelta Barbero JM, Cho RI, Otto BA, Carrau RL, et al: Comparative analysis of the exposure and surgical freedom of the endoscopic extended minipterional craniotomy and the transorbital endoscopic approach to the anterior and middle cranial fossae. Oper Neurosurg (Hagerstown) 17:174-181, 2019

23. Oshiro EM, Rini DA, Tamargo RJ: Contralateral approaches to bilateral cerebral aneurysms: a microsurgical anatomical study. J Neurosurg 87:163-169, 1997

24. Ottenhausen M, Rumalla K, Alalade AF, Nair P, La Corte E, Younus I, et al: Decision-making algorithm for minimally invasive approaches to anterior skull base meningiomas. Neurosurg Focus 44(4):E7, 2018

25. Park HH, Sung KS, Moon JH, Kim EH, Kim SH, Lee KS, et al: Lateral supraorbital versus pterional approach for parachiasmal meningiomas: surgical indications and esthetic benefits. Neurosurg Rev 43:313-322, 2020

26. Perneczky A, Fries G: Endoscope-assisted brain surgery: part 1 -evolution, basic concept, and current technique. Neurosurgery 42:219-225, 1998
27. Reisch R, Perneczky A: Ten-year experience with the supraorbital subfrontal approach through an eyebrow skin incision. Neurosurgery 57 (4 Suppl):242-255, 2005

28. Rychen J, Croci D, Roethlisberger M, Nossek E, Potts M, Radovanovic I, et al: Minimally invasive alternative approaches to pterional craniotomy: a systematic review of the literature. World Neurosurg 113:163-179, 2018

29. Rychen J, Croci D, Roethlisberger M, Nossek E, Potts MB, Radovanovic I, et al: Keyhole approaches for surgical treatment of intracranial aneurysms: a short review. Neurol Res 41:68-76, 2019

30. Tra H, Huynh T, Nguyen B: Minipterional and supraorbital keyhole craniotomies for ruptured anterior circulation aneurysms: experience at single center. World Neurosurg 109:36-39, 2018

31. Tullos HJ, Conner AK, Baker CM, Briggs RG, Burks JD, Glenn CA, et al: Mini-pterional craniotomy for resection of parasellar meningiomas. World Neurosurg 117:e637-e644, 2018

32. van Lindert E, Perneczky A, Fries G, Pierangeli E: The supraorbital keyhole approach to supratentorial aneurysms: concept and technique. Surg Neurol 49:481-490, 1998

33. Wang J, Xu F: Unilateral supraorbital approach to clip bilateral posterior communicating artery aneurysms: 2-dimensional operative video. World Neurosurg 119:256, 2018

34. Welling LC, Figueiredo EG, Wen HT, Gomes MQT, BorSeng-Shu E, Casarolli C, et al: Prospective randomized study comparing clinical, functional, and aesthetic results of minipterional and classic pterional craniotomies. J Neurosurg 122:1012-1019, 2015

35. Yasargil MG, Fox JL: The microsurgical approach to intracranial aneurysms. Surg Neurol 3:7-14, 1975

\section{Disclosures}

Dr. Prevedello is a consultant for Integra LifeSciences Corp., Stryker Corp., and Medtronic Corp. He has equity in 3 Rivers LLC, eLUM Technologies LLC, and Soliton LLC. He also receives royalties from KLS-Martin and Mizuho.

\section{Author Contributions}

Conception and design: Martínez-Pérez, Albonette-Felicio. Acquisition of data: Martínez-Pérez, Albonette-Felicio. Analysis and interpretation of data: Martínez-Pérez, Albonette-Felicio. Drafting the article: Prevedello, Martínez-Pérez. Critically revising the article: Prevedello, Martínez-Pérez, Hardesty. Reviewed submitted version of manuscript: Martínez-Pérez. Statistical analysis: Martínez-Pérez, Hardesty. Administrative/ technical/material support: Martínez-Pérez, Albonette-Felicio. Study supervision: Prevedello, Hardesty.

\section{Correspondence}

Daniel M. Prevedello: The Ohio State University, Wexner Medical Center, Columbus, OH. daniel.prevedello@osumc.edu. 